\title{
Thermal environment adaptability design of space-based infrared imaging system
}

\author{
Qinglong $\mathrm{Hu}^{1,2^{*}}$, Guangwei Shi ${ }^{1}$, Fan Jiang ${ }^{1}$, Huaide Zhou ${ }^{1}$, Zhaohui Li ${ }^{1}$, Liang Yang ${ }^{1}$, Xuejun Zhang ${ }^{1}$ \\ ${ }^{1}$ Changchun Institute of Optics, Fine Mechanics and Physics, Chinese Academy of Sciences, Changchun 130033, China \\ ${ }^{2}$ University of Chinese Academy of Sciences, Beijing 100049, China
}

Corresponding Author Email: huqinglong.hit@163.com

https://doi.org/10.18280/ijht.360139

Received: 17 July 2017

Accepted: 8 October 2017

\section{Keywords:}

space-based, infrared imaging, thermal environment, non-thermal design, thermal control

\begin{abstract}
This study discusses the influence of temperature on infrared optical system and introduced the principle and method of non-thermal design. Based on the requirements of orbital thermal environment and technical indexes of a space-based infrared imaging system, this study conducts the thermal environment adaptability design of an imaging system. The nonthermal design of the optical system is carried out by combining active and passive methods, and thermal design and thermal analysis are carried out for the thermal environment of space orbit in which the system works. Finally, the final imaging system is validated by the thermal imaging experiment. The experimental results show that the system can eliminate the influence of temperature change on image quality in the temperature range of $-20 \sim+50{ }^{\circ} \mathrm{C}$, and can adapt to the thermal environment of space orbit. The imaging quality can meet the application requirements under different temperature conditions.
\end{abstract}

\section{INTRODUCTION}

With the development of infrared technology, the requirements of infrared imaging system for thermal environment adaptability becomes higher and higher. In particular, satellite-borne, missile-borne and airborne infrared systems are often required to operate in a large temperature range. Under different temperature conditions, due to the thermal effect of optical materials and mechanical materials, some parameters of the optical system change correspondingly, and then the optimal image plane of the system deviates, and the image quality is reduced to blurred image and lower contrast, which finally affect the performance of the infrared imaging system.

In order to improve the environmental adaptability of the system, the optical system is usually required to maintain good imaging quality in a wide temperature range during the design of the optical and mechanical system. With the change of temperature, the refractive index of the optical materials, the size and the surface shape of the optical elements will change [1], which will cause defocus and other aberrations of the optical system, leading to poor imaging quality. Compared with visible optical materials, infrared optical materials have high refractive index temperature variation coefficient, so the influence of temperature variation on the performance of infrared optical system is more serious [2]. Therefore, in order to ensure the stable operation of the infrared optical system in a wide temperature range, it is necessary to eliminate the influence of temperature on the infrared optical system, that's, non-thermal design of infrared optical system.

For the space-based infrared imaging system, its environment is complex, and the space vacuum environment cannot carry out convection heat dissipation, so when the sun directly illuminates the remote sensor, its temperature can reach above $100^{\circ} \mathrm{C}$ if not protected; when the spacecraft enters the shadow zone, the temperature will be lower than $100^{\circ} \mathrm{C}$. Obviously, it's a fatal impact of such a large temperature difference on the space-based infrared imaging system. Therefore, it is required to control the working temperature of the space-based infrared imaging system within the temperature range in which it can work normally by thermal control means.

This study firstly introduces the influence of temperature on infrared optical system and then the principle and method of non-thermal design. Based on the requirements of orbital thermal environment and technical indexes of a space-based infrared imaging system, this study conducts the thermal environment adaptability design of an imaging system. The non-thermal design of the optical system is carried out by combining active and passive methods, and thermal design and thermal analysis are carried out for the thermal environment of space orbit in which the system works. Finally, the final imaging system is validated by the thermal imaging experiment. The experimental results show that the system can eliminate the influence of temperature change on image quality in the temperature range of $-20 \sim+50^{\circ} \mathrm{C}$, and can adapt to the thermal environment of space orbit. The imaging quality can meet the application requirements under different temperature conditions.

\section{INFLUENCE OF TEMPERATURE ON INFRARED OPTICAL SYSTEM}

\subsection{The refractive index of optical elements varies with temperature}

The refractive index of the optical materials varies with temperature, which changes the focal length of the lens or optical system. The temperature coefficient of infrared optical materials is much larger than that of ordinary optical 
glass, for example, the typical value $\mathrm{dn} / \mathrm{dt}$ of germanium monocrystalline is about $396 \times 10^{-6} \mathrm{C}^{-1}$, while the temperature coefficient of $\mathrm{K} 9$ glass is only $2.8 \times 10^{-6}{ }^{\circ} \mathrm{C}^{-1}$. Therefore, the influence of temperature on refractive index is particularly obvious in infrared system [3].

\subsection{Radius of curvature and center thickness of optical elements vary with temperature}

This is caused by expanding with heat and contracting with cold of the component materials, which is related to the linear expansion coefficient $\mathrm{a}_{0}$ of the optical materials. When the temperature changes, the radius of curvature and the central thickness of the optical element become:

$\mathrm{D}^{\prime}=\mathrm{D}+\mathrm{dD}=\mathrm{D}+\mathrm{D} \cdot \mathrm{a}_{0} \cdot \mathrm{dT}$

$\mathrm{R}^{\prime}=\mathrm{R}+\mathrm{dR}=\mathrm{R}+\mathrm{R} \cdot \mathrm{a}_{0} \cdot \mathrm{dT}$

where, R, D and $\mathrm{R}^{\prime}, \mathrm{D}^{\prime}$ are curvature radius and central thickness before and after temperature change, respectively. and $\mathrm{dT}$ is temperature change.

\subsection{Thermal effect of the supporting structure materials}

As the temperature changes, the size of the supporting structure will also change, which will cause a change in the spacing of the optical elements, and thereby affect the imaging quality. This is related to the linear expansion coefficient of the supporting structure materials. When the temperature changes, the length of the lens tube material changes as follows:

$\mathrm{L}^{\prime}=\mathrm{L}+\mathrm{dL}=\mathrm{L}+\mathrm{L} \cdot \mathrm{a}_{0} \cdot \mathrm{dT}$

where, $\mathrm{L}$ and $\mathrm{L}^{\prime}$ are the length of the supporting structure before and after the temperature change. and $\mathrm{dT}$ is temperature change.

\section{METHOD OF NON-THERMAL DESIGN}

According to the research reports both at home and abroad, there are three methods to carry out non-thermal design of infrared optical system: mechanical passive, electronic active and optical passive [4-10].

\subsection{Mechanical passive}

Mechanical passive is to enable one or a group of lenses to generate axial displacement by the use of temperaturesensitive mechanical materials or memory alloys, so as to compensate for the image plane displacement caused by the temperature changes. This method needs to calculate the position of the optimal image plane at different temperature and compensate the displacement of the optimal image plane by different amount of metal expansion according to the displacement of the optimal phase plane. The method cannot correct the aberration imbalance caused by thermal effect. Even it can compensate the movement of the optimal image plane, it is difficult to maintain the original image quality. This method additionally adds mechanical compensation components so that the volume of the system becomes larger and the mass increases.

\subsection{Electronic active}

The electronic active uses the temperature sensor to detect the temperature change, then calculates the image plane displacement caused by the temperature change, and drives the lens to generate the axial displacement by virtue of electric machinery to achieve the compensation effect. This method adopts a large number of temperature sensors, so as to process the gradient change of the system's temperature, and accurately solve the relation between the temperature and the image plane displacement.

\subsection{Optical passive}

In brief, the optical passive non-thermal design is to utilize the difference between the thermal characteristics of optical materials, eliminate the influence of temperature through reasonable combination of different characteristic materials, and reasonably match the optical power distribution scheme of the materials, so that the defocus of the optical system resulting from the change in temperature offsets the defocus of the the mechanical tube resulting from expanding with heat and contracting with cold, realizing the purpose of automatic temperature compensation. Strictly speaking, the non-thermal design of the optical system needs to meet the requirements of thermal elimination, achromatic aberration and optical power [9-22].

The optical power changes caused by the dispersion of the optical elements in different spectral sections of the optical system are as follows:

$\Delta \mathrm{f}_{\mathrm{c}}=\left(\frac{1}{\mathrm{~h}_{1} \phi}\right)^{2} \sum_{\mathrm{i}=1}^{\mathrm{k}}\left(\mathrm{h}_{\mathrm{i}}^{2} \omega_{\mathrm{i}} \emptyset_{\mathrm{i}}\right)$

where, $h_{i}$ is the paraxial ray projection height of the ith lens, $\emptyset_{\mathrm{i}}$ is lens optical power and $\omega_{\mathrm{i}}$ is dispersion factor.

The change in focal length of the optical system caused by the change in temperature of the optical element is:

$$
\Delta f_{T}=\left(\frac{1}{h_{1} \varnothing}\right)^{2} \int\left(h_{i}^{2} \alpha_{g i} \chi_{i} \emptyset_{i}\right) \mathrm{dT}
$$

where, $\chi_{1}$ is normalized thermal differential system and $\chi_{1}=$ $\frac{\mathrm{dn}}{\mathrm{n}-1}-\alpha_{\mathrm{gi}}, \alpha_{\mathrm{gi}}$ is linear expansion coefficient of the lens.

The non-thermal design must meet the requirements of focal power, correction of chromatic aberration and athermalization, that's, the system must satisfy the following non-thermal equation:

$$
\begin{aligned}
& \emptyset=\frac{1}{h_{1}} \sum_{i=1}^{k} h_{i} \emptyset_{i} \\
& \Delta f_{c}=\left(\frac{1}{h_{1} \varnothing}\right)^{2} \sum_{i=1}^{k}\left(h_{i}^{2} \omega_{i} \emptyset_{i}\right)=0 \\
& \Delta f_{T}=\left(\frac{1}{h_{1} \varnothing}\right)^{2} \int\left(h_{i}^{2} \alpha_{g i} \chi_{i} \emptyset_{i}\right) \mathrm{dT}=\alpha_{m} L
\end{aligned}
$$

where, $\alpha_{\mathrm{m}}$ is linear expansion coefficient of system structural element material and $\mathrm{L}$ is the length of the system structure. With the above calculation, the system paraxial relation that satisfies the requirement of non-thermal optical system can be obtained, and the initial structure of the optical system can be obtained by matching the material of the lens tube and the material of the transmission element. 


\section{THERMAL ENVIRONMENT ADAPTABILITY DESIGN OF INFRARED IMAGING SYSTEM}

\subsection{Optical design index and input condition}

(1) Working wavelength: $3.7 \mu \mathrm{m} \sim 4.8 \mu \mathrm{m}$.

(2) Focal length: $884 \mathrm{~mm}$

(3) Relative aperture: 1:4.

(4) Instantaneous field of view: $0.96^{\circ} \times 0.76^{\circ}$.

(5) Type of orbit: Sun-synchronous orbit (Orbit height: $400 \mathrm{~km})$;

(6) Spacecraft temperature environment: $-20^{\circ} \mathrm{C} \sim 50^{\circ} \mathrm{C}$.

\subsection{Optical system design}

The optical system is divided into afocal optical path and imaging optical path with parallel optical path as the boundary. In order to balance the aberration sufficiently, the optical system design adopts the design idea of active and passive non-thermal technology, that's, the afocal optical path adopts the passive non-thermal design, and the imaging optical path adopts the focusing mode to realize the active non-thermal. The afocal optical path consists of the primary mirror, the secondary mirror and the collimating lens, and the imaging optical path consists of focusing lenses and the relay lenses. The basic imaging principle of the optical system is that the target heat radiation reaches the primary mirror, reflects the first image plane through the primary mirror and the secondary mirror and then passes through the collimating lens to form the condensed parallel light with the exit pupil. The condensed parallel light passes through the focusing group and then the relay lenses to reach the detector. As shown in Figure 1 for the structure of optical path, all optical elements of the optical path after the collimating lens are arranged on the optics bench which is on the back of the primary mirror.

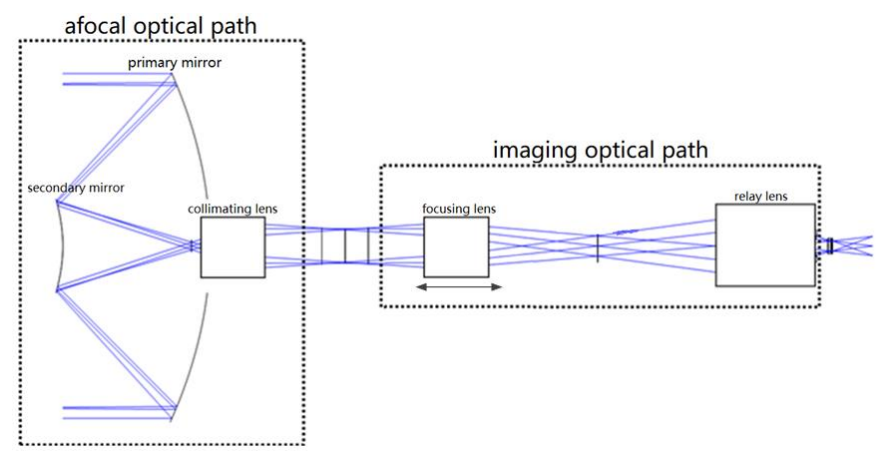

Figure 1. Optical system structure

\subsubsection{Afocal optical path design}

Technical index of the afocal optical path implemented as follows:

(1) Angular magnification: $-10^{\times}$.

(2) Entrance pupil diameter: $\Phi 221 \mathrm{~mm}$.

(3) Object-wide field of view: $1.05^{\circ} \times 0.95^{\circ}$.

(4) Distortion: less than $0.023 \%$.

The field curvature and distortion curves of the afocal optical path are shown in Figure 2; the optical transfer function curve is shown in Figure 3 (central shading is not taken into account), the image-side corresponding Nyquist frequency is $1.86 \mathrm{lp} / \mathrm{mrad}$, and the imaging quality is diffraction limit.

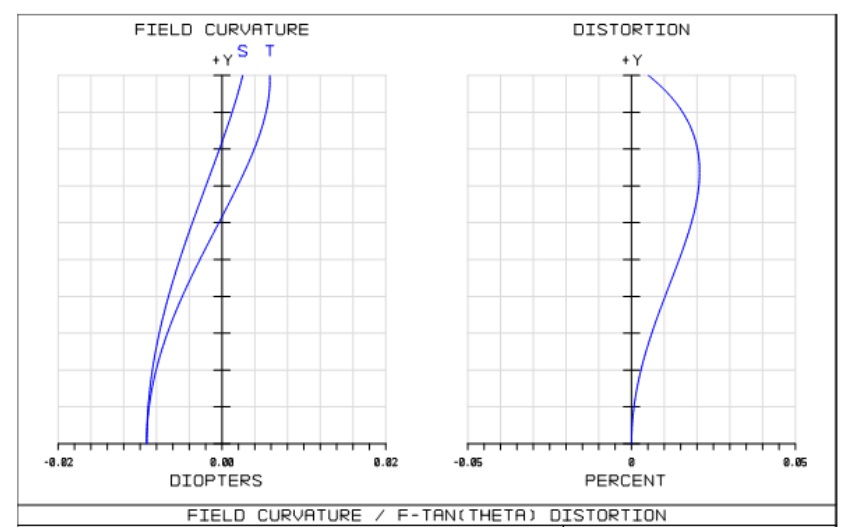

Figure 2. Field curvature and distortion curves of the afocal optical path

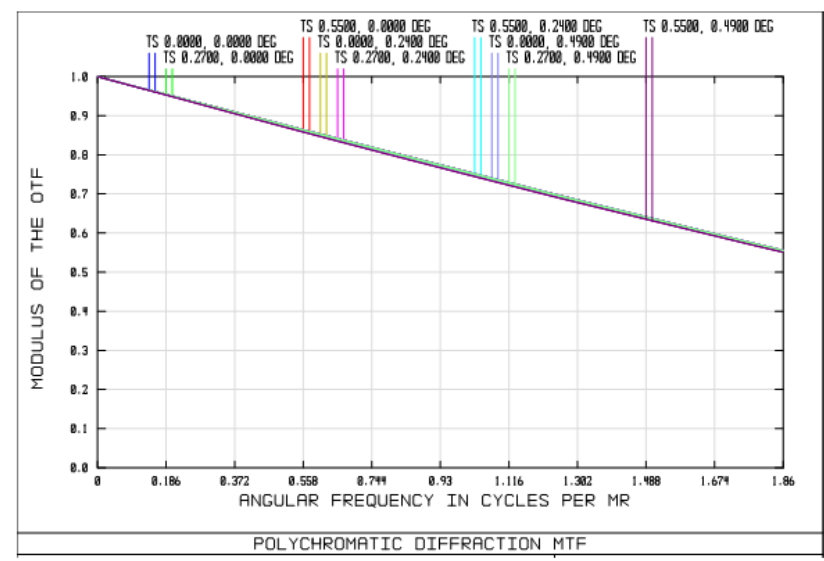

Figure 3. Optical transfer function curve of the afocal optical path

4.2.2 Joint design of imaging optical path and afocal optical path

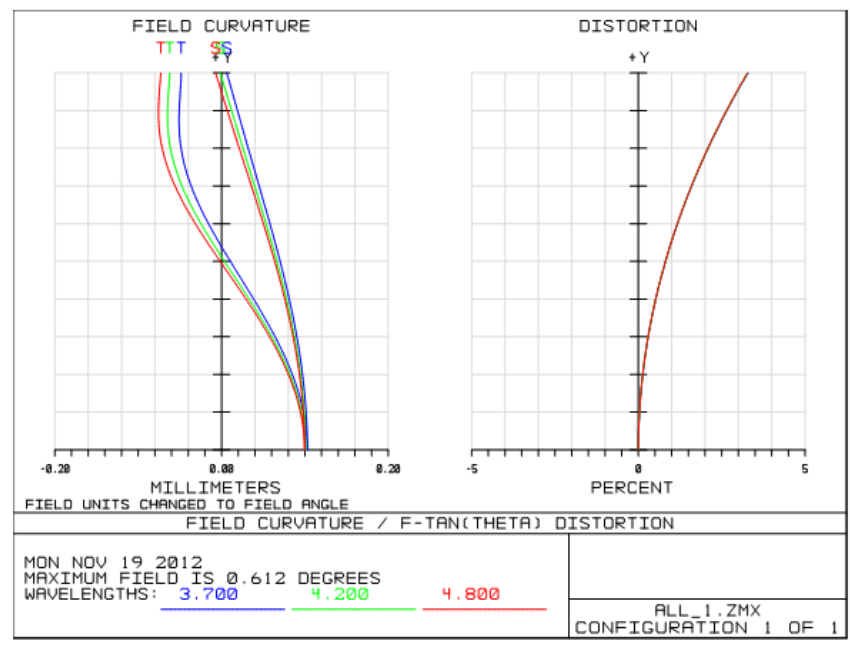

Figure 4. Field curvature and distortion curves of the joint design of the two optical paths 


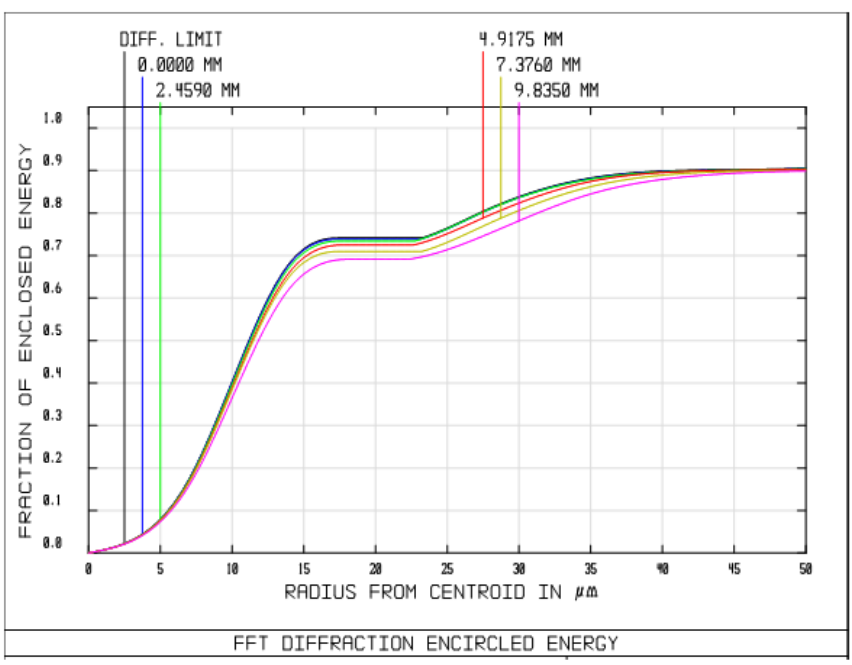

Figure 5. Energy concentration curve of the joint design of the two optical paths

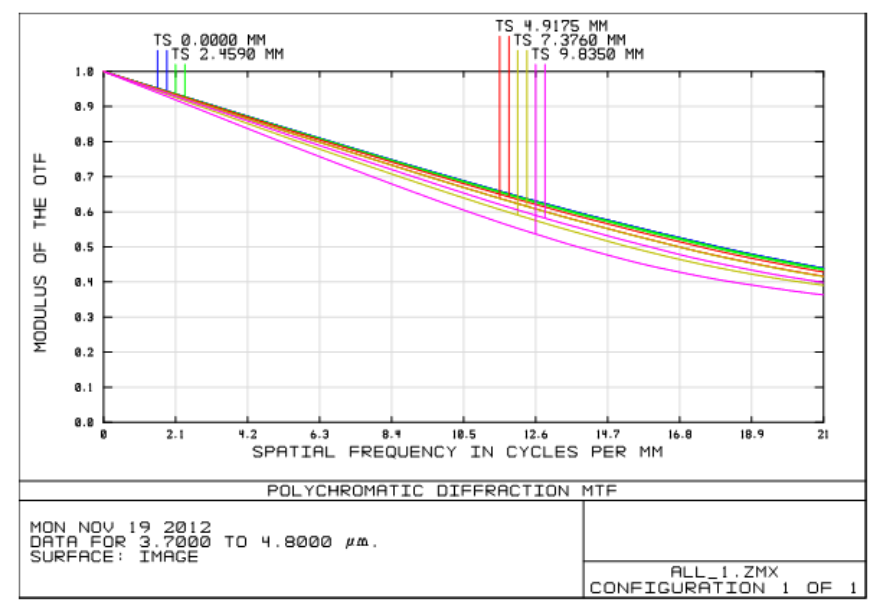

Figure 6. Optical transfer function curve of the joint design of the two optical paths

The imaging optical path is completed on the basis of the afocal optical path design, and the technical indexes are realized after the joint design of the two optical paths as follows:

(1) Focal length: $884 \mathrm{~mm}$.

(2) Relative aperture: 1:4.

(3) Object-wide field of view: $0.96^{\circ} \times 0.76^{\circ}$.

(4) Spectral range: $3.7 \mu \mathrm{m} \sim 4.8 \mu \mathrm{m}$.

(5) Distortion: $3.3 \%$.

The field curvature and distortion curves of the joint design of the two optical paths are shown in Figure 4; the energy concentration curve is shown in Figure 5; as shown in Figure 6 for the optical transfer function curve, the imageside corresponding Nyquist frequency is $21 \mathrm{lp} / \mathrm{mrad}$, and the system design reaches the diffraction limit.

\subsection{Influence of non-thermal design on focusing quantity}

In order to balance the aberration sufficiently, the optical system design adopts the design idea of the combination of the active and passive non-thermal technology, that's, the afocal optical path adopts the passive non-thermal design, and the imaging optical path adopts the focusing mode to realize the active non-thermal. This section will give the influence of passive non-thermal design of afocal optical path on the active non-thermal design of the imaging optical path, that's, the influence on the focusing amount.

When the temperature of the optical system changes, the radius, space, thickness and refractive index of each plane will change, so that the image plane will defocus. If the defocus amount exceeds the focal depth range, the imaging quality of the system will inevitably be seriously influenced, so the system needs to be focused. In the initial scheme, the primary and secondary mirrors and the support of primary and secondary mirrors in the afocal optical path are made of aluminum alloy. The system is analyzed according to the material selection and design model of the optical machine system. Table 1 shows the defocus amount and the movement amount of the focusing group at different temperatures.

Table 1. Movement amount of the focusing group at different temperatures

\begin{tabular}{lccccccccc}
\hline $\begin{array}{c}\text { Temperature } \\
\left.\text { ( }{ }^{\circ} \mathrm{C}\right)\end{array}$ & -30 & 0 & 10 & 15 & 20 & 25 & 30 & 40 & 60 \\
\hline $\begin{array}{c}\text { Focusing } \\
\text { quantity(mm) }\end{array}$ & -0.64 & -0.25 & -0.12 & -0.05 & 0 & 0.06 & 0.15 & 0.28 & 0.54 \\
\hline $\begin{array}{c}\text { Defocusing } \\
\text { amount(mm) }\end{array}$ & 0.55 & 0.21 & 0.10 & 0.04 & 0 & -0.07 & -0.12 & -0.23 & -0.45 \\
\hline
\end{tabular}

The focal depth of the optical system is $\pm 0.13 \mathrm{~mm}$. It can be seen from Table 1 that when the temperature change is \pm $10{ }^{\circ} \mathrm{C}$, the focusing amount is within the focal depth and needs no focusing. When the temperature is larger than this temperature range, the focusing amount is the largest of $0.64 \mathrm{~mm}$.

By optimizing the linear expansion coefficient of the supporting structure of the secondary mirror, the passive nonthermal design of the afocal optical path can be realized to some extent. In particular, with the initial structure as the basis and the linear expansion coefficient of supporting material of the secondary mirror as a variable, the material selection and size parameters of the collimating lens are jointly calculated to obtain that the temperature adaptability of the afocal optical path can be improved when the secondary mirror supporting material is copper alloy.

The temperature defocus of the entire optical system is recalculated according to the modified structural material, with the results shown in Table 2 . It can be seen that when the temperature variation of the optical system is within \pm 20 ${ }^{\circ} \mathrm{C}$ after the non-thermal design, the defocus amount does not exceed the depth of focus, and the maximum focusing amount is $0.26 \mathrm{~mm}$, indicating that the environmental adaptability of the system is improved greatly. After the nonthermal design, the optical transfer function of the afocal optical path at $-30^{\circ} \mathrm{C}$ and $+60^{\circ} \mathrm{C}$ is as shown in Figure 7 . It can be seen that the afocal optical path after the non-thermal has the imaging quality of diffraction limit in a wide temperature range.

Table 2. A focus amount at different temperatures after optimization of the system

\begin{tabular}{ccccccccc}
\hline Temperature $\left({ }^{\circ} \mathrm{C}\right)$ & -30 & -10 & 0 & 10 & 20 & 30 & 40 & 60 \\
\hline $\begin{array}{c}\text { Defocusing } \\
\text { amount(mm) }\end{array}$ & -0.26 & -0.16 & -0.11 & -0.05 & 0 & -0.05 & -0.11 & -0.22 \\
\hline
\end{tabular}




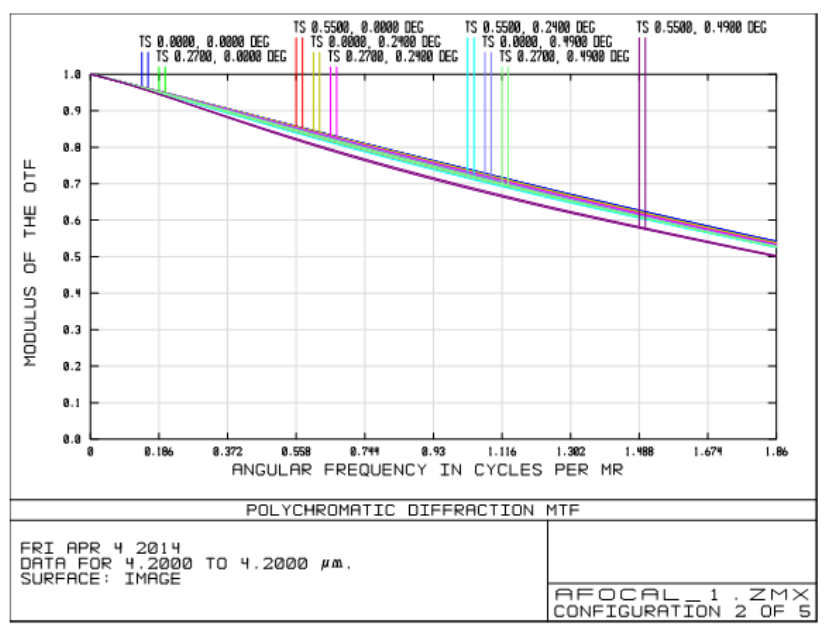

(a) System's environment temperature is $-30^{\circ} \mathrm{C}$

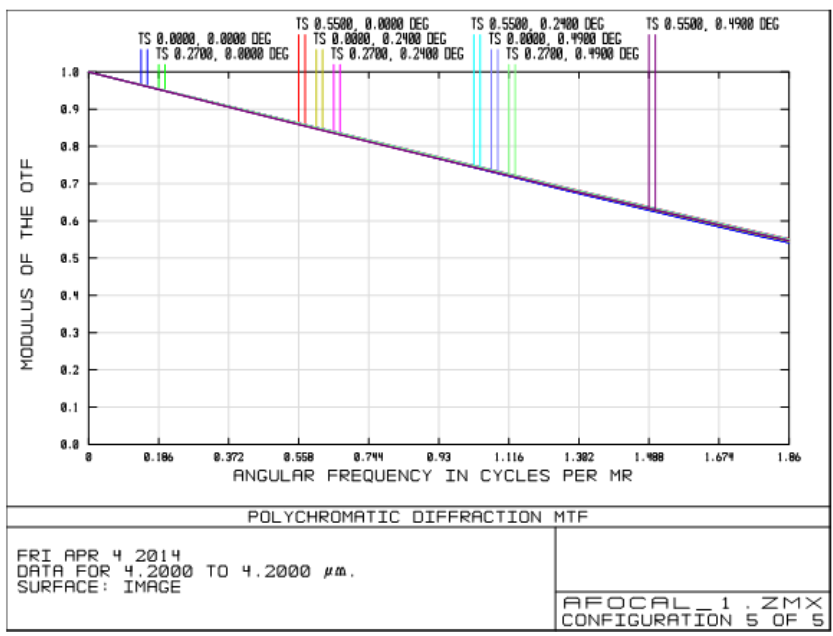

(b) System's environment temperature is $+60^{\circ} \mathrm{C}$

Figure 7. Optical transfer function of afocal optical path with a non-thermal design

\subsection{Design and analysis of thermal control}

\subsubsection{Design input and temperature control index}

The space-based infrared imaging system in this study works in the $400 \mathrm{~km}$ sun-synchronous orbit, ascending node time is $18: 00$, its body is installed in the satellite platform cabin, and the lens is installed with heat dissipation tube outside the cabin to observe the earth. The temperature in the cabin is controlled by the satellite platform at $-20{ }^{\circ} \mathrm{C} \sim+$ $50{ }^{\circ} \mathrm{C}$.

In order to obtain better imaging effect, active thermal control and passive thermal control are used to control the whole temperature level of infrared imaging system at $0 \sim 30^{\circ}$ and the temperature difference of primary and secondary mirrors at $4^{\circ} \mathrm{C}$.

\subsubsection{Thermal control design}

In this study, active thermal control and passive thermal control are used in thermal control design.

Passive thermal control measures are introduced as follows: the outer surface of infrared imaging system is coated with multi-layer insulating material; the mounting point of the satellite platform is installed with a $10 \mathrm{~mm}$ polyimide thermal pad; the surface of the optical system mounting substrate is subject to black anodic oxidation treatment; heat dissipation tube and its surrounding, back of secondary mirror, secondary mirror support and external frame use ITO polyimide secondary surface mirror, and the other multi-layers all use $25 \mu$ double-sided aluminized film as mask; the devices with high power consumption should be installed with the optics bench for thermal conduction, heat conduction path should be reasonable planed, the heat conduction installation of the imaging unit and the refrigerator is shown in Figure 8, in which the working heat consumption of the imaging unit is conducted to the optics bench through the mounting bracket, the refrigerator is conducted to the optics bench through the heat conduction filler, both of which are finally conducted out through a heat dissipation tube installed with thermal conduct of optics bench; thermally conductive and installed with the optics bench; thermal cover and the optics bench mounting surface are coated with thermal grease.

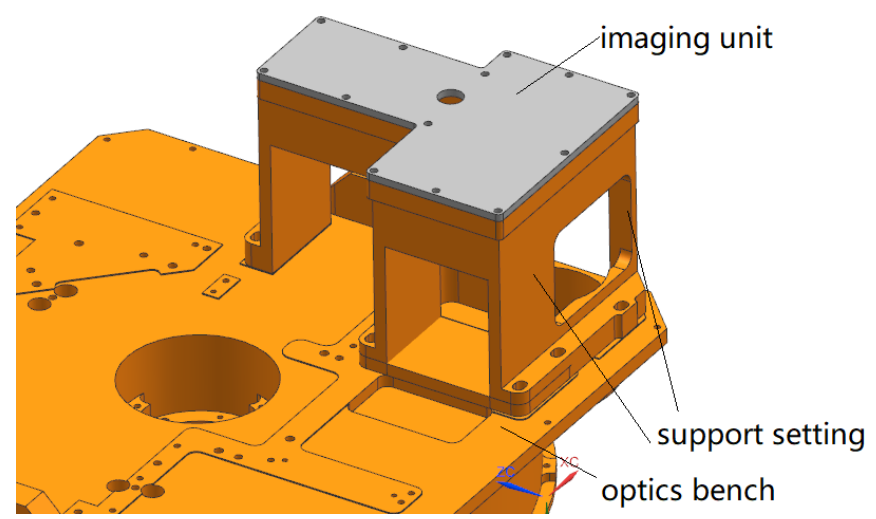

(a) Heat conduction of imaging unit

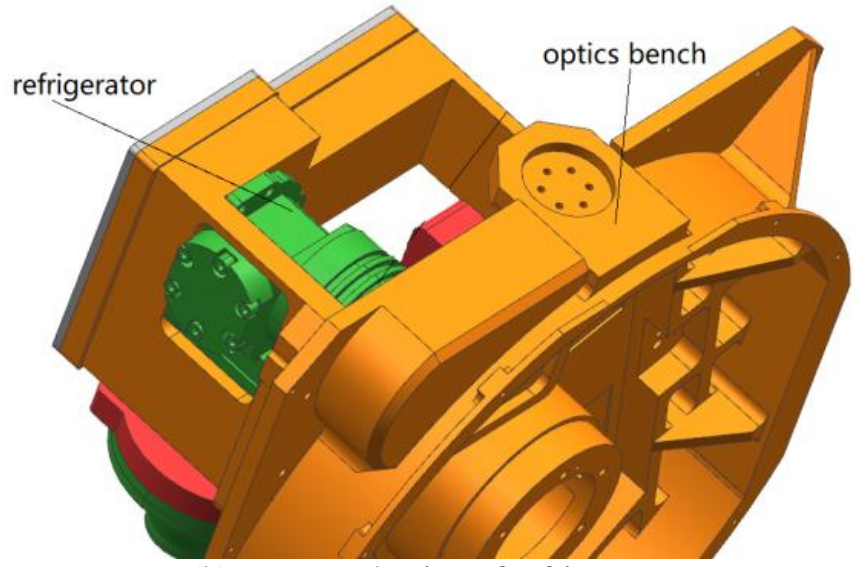

(b) Heat conduction of refrigerator

Figure 8. Heat conduction installation of the imaging unit and the refrigerator with optics bench

Based on the passive thermal control measures, the active thermal control design is introduced as follows: in the infrared image system, three active heating circuits are set, and the positions of the heating circuits are respectively at heat dissipation cover, secondary mirror and its supporting setting, and the protection rear cover of imaging optical path, as shown in Figure 9; three active heating temperature control points are set in close-circuit, using MF501 thermistor for temperature measurement, 3-path temperature control point positions: the back of the primary mirror, the back of the secondary mirror, and the outside of heat dissipation tube. 


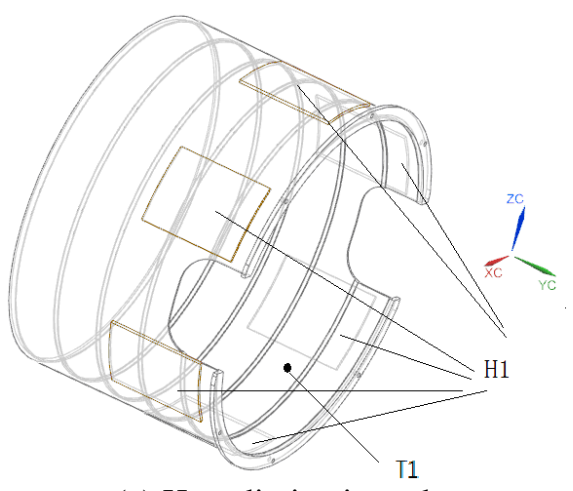

(a) Heat dissipation tube

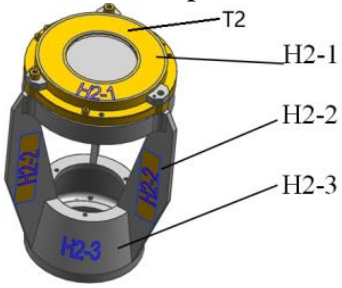

(a) Secondary mirror and its supporting setting

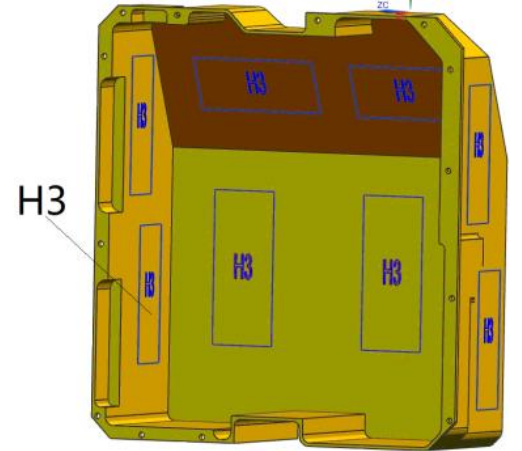

(a) Protection rear cover of imaging optical path

Figure 9. Position of active thermal control heating circuit

\subsubsection{Thermal analysis}

According to the thermal design scheme in 4.4.2, and in combination with the product model and orbit parameters, thermal analysis of the product under extreme high temperature conditions and extreme low temperature conditions is carried out. The analytic results are shown in Figures 10 and 11 .

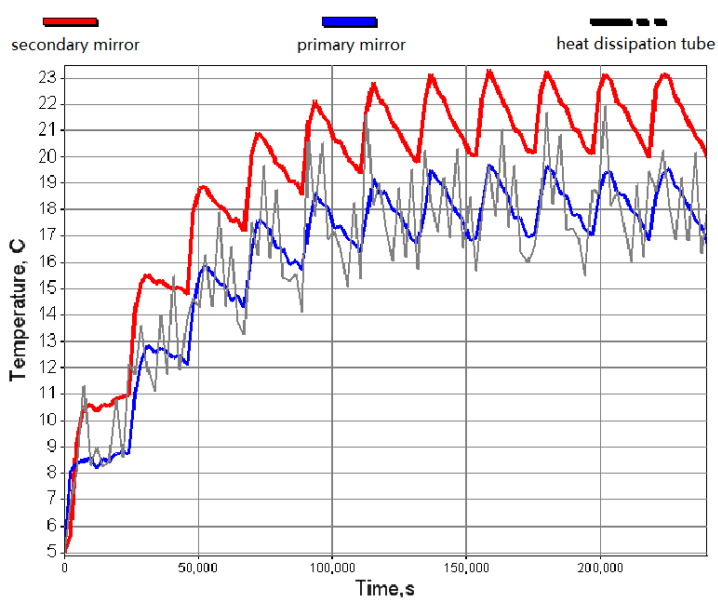

Figure 10. Simulative analysis results of extremely high temperature conditions

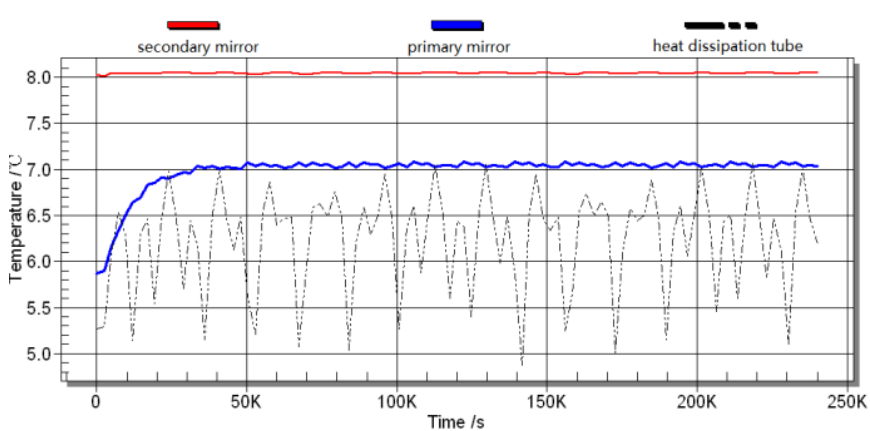

Figure 11. Simulative analysis results of extremely low temperature condition

From Figure 10, it can be seen that the temperature of the primary mirror fluctuates in the range of $20.0^{\circ} \mathrm{C}$ to $23.2^{\circ} \mathrm{C}$ when the extremely high temperature condition is finally balanced; the temperature of the secondary mirror fluctuates from $16.8^{\circ} \mathrm{C}$ to $19.8^{\circ} \mathrm{C}$; due to the influence of external heat flow, the temperature of heat dissipation cover is $15.5^{\circ} \mathrm{C} \sim 21.9^{\circ} \mathrm{C}$. The temperature difference between the primary mirror and the secondary mirror shall not exceed $3.5^{\circ} \mathrm{C}$ from the beginning of temperature rise to final equilibrium. It can be seen from Figure 11 that at the final equilibrium of the extremely low temperature condition, the primary mirror temperature is about $7^{\circ} \mathrm{C}$, the secondary mirror temperature is about $8^{\circ} \mathrm{C}$, and the heat dissipation cover temperature is about $6^{\circ} \mathrm{C}$. From the beginning of temperature rise to final equilibrium, the temperature difference between the primary mirror and the secondary mirror shall always not exceed $2.5^{\circ} \mathrm{C}$, and after equilibrium, the temperature difference between the primary and secondary mirrors shall be about $1^{\circ} \mathrm{C}$. In summary, the results of thermal analysis meet the requirements of thermal control design.

\section{EXPERIMENT VERIFICATION}

The designed infrared imaging system and other components of the system are tested for high and low temperature imaging. An independent cabinet is designed to simulate the installation cabin of the equipment on the orbit. The internal temperature of the cabinet simulates the thermal boundary condition on the orbit, i.e. the temperature condition of $-20 \sim+50^{\circ} \mathrm{C}$. The thermal imaging experiment site of the system is shown in Figure 12.

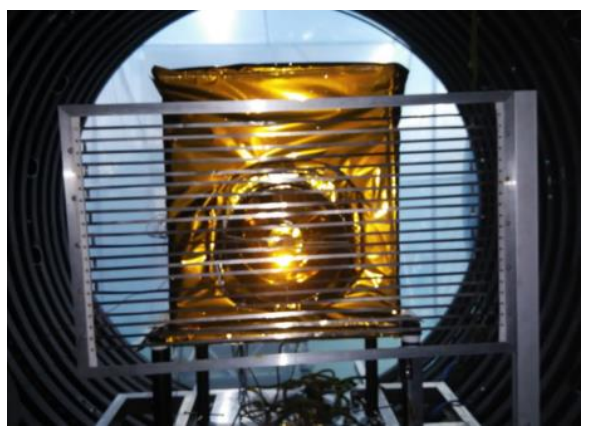

Figure 12. Thermal imaging experiment site of the system 
During the experiment, the target is imaged under the conditions of low temperature and high temperature respectively. Before imaging, the system is held at the temperature point for two hours, then the system is turned on for imaging, and the collected image is shown in Figure 13. Through thermal imaging experiment, the rationality and effectiveness of optical design, non-thermal design and thermal control design of infrared imaging system are verified in this study.

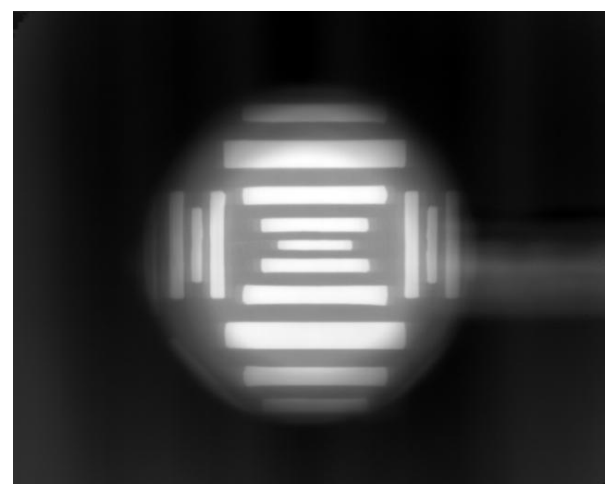

(a) Imaging at $-20^{\circ} \mathrm{C}$

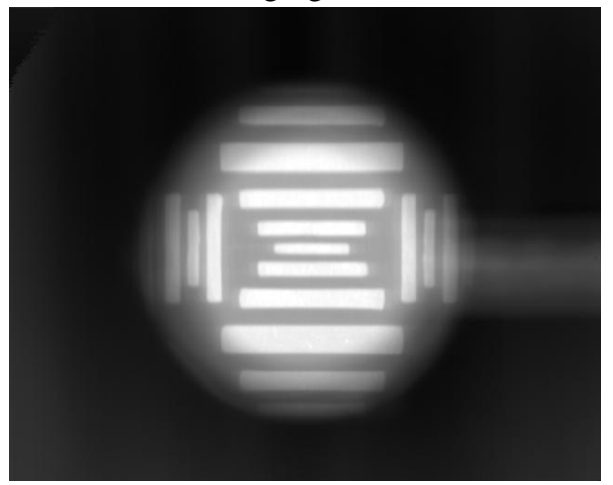

(b) Imaging at $+20^{\circ} \mathrm{C}$

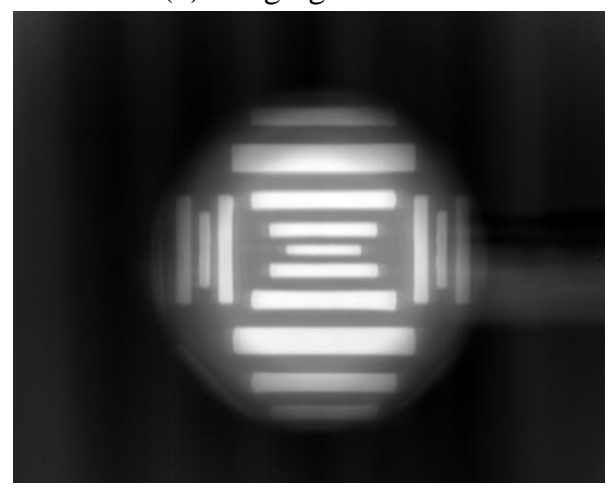

(c) Imaging at $+50^{\circ} \mathrm{C}$

Figure 13. Imaging effect of different temperatures

\section{CONCLUSIONS}

This study discusses the influence of temperature on infrared optical system and introduces the principle and method of non-thermal design. Based on the requirements of orbital thermal environment and technical indexes of a spacebased infrared imaging system, this study conducts the thermal environment adaptability design of an imaging system. The non-thermal design of the optical system is carried out by combining active and passive methods, and thermal design and thermal analysis are carried out for the thermal environment of space orbit in which the system works. Finally, the final imaging system is validated by the thermal imaging experiment. The experimental results show that the system can eliminate the influence of temperature change on image quality in the temperature range of -20 + $50{ }^{\circ} \mathrm{C}$, and can adapt to the thermal environment of space orbit. The imaging quality can meet the application requirements under different temperature conditions.

\section{REFERENCES}

[1] Xi X, Cen ZF, Li XT. (2005). Application of athermalisation in optical systems. Infrared and Laser Engineering 34(4): 388-390. https://doi.org/10.3969/j.issn.1007-2276.2005.04.003.

[2] Shen HH, Wang GH, Ding JW, et al. (2010). Application of active-athermal compensation to airborne IR optical systems. Optics and Precision Engineering 18(3): 593-601.

[3] Marcin les'niewski. (1997). Material problem in athermalization of optical systems. Optical Engineering 36(6): 1596.

[4] Pilkington. (1986). Athermalization techniques in infrared systems. SPIE 0655: 142-153.

[5] Liu L. (2010). Study on fast speed optical system of uncooled MWIR imager. Soochow: Soochow University, China. https://doi.org/10.7666/d.y1733357.

[6] Wu XJ, Meng JH. (2003). Approach of athermalizing infrared optical systems. Infrared and Laser Engineering 32(6): 572-576. https://doi.org/10.3969/j.issn.10072276.2003.06.006.

[7] Li H, Han WQ, Shen MZ. (2009). Passive athermal design and measurement of MWIR optical system. Infrared and Laser Engineering 38(4): 687-691. https://doi.org/10.3969/j.issn.1007-2276.2009.04.024.

[8] Hu YX, Zhou SX, Yang JF, et al. (2000). Opticalmechnical-thermal co-design of infrared systems. Infrared Technology 22(2): 32-35. https://doi.org/10.3969/j.issn.1001-8891.2000.02.007

[9] Song D. (2017). Visualization of information. NeuroQuantology 16(1): 32-36. https://doi.org/10.14704/nq.2018.16.1.1131.

[10] Doruk OR. (2017). Control of hopf bifurcations in hodgkin-huxley neurons by automatic temperature manipulation. NeuroQuantology 16(1): 59-74. https://doi.org/10.14704/nq.2018.16.2.1140.

[11] Rayces JL. (1990). Thermal compensation of infrared achrcmatic objectives with three optical materials. SPIE, 1354: 752-759.

[12] Guo Yonghong, Shen Mangzuo. (2000). Athermal design for infrared diffractive/refractive optical system. Acta Optic Sinica 20(10): 1392-1395. https://doi.org/10.3321/j.issn:0253-2239.2000.10.017.

[13] Qu HM, Zhang X, Wang LJ, et al. (2012). Design of a low f-number compact athermalizing infrared optical system. Acta Optic Sinica 32(3): 0322003-1-8.

[14] Tamagawa Y, Tajime T. (1996). Expansion of an athermal chart into a multilens system with thick lenses spaced apart. Opt Eng 35(10): 3001-3006.

[15] Yu Y, Jin YP, Pan ZX, et al. (2010). Athermal design of cooled LWIR optical system with extensive temperature range. Infrared Technology 33(10): 585-587. https://doi.org/10.3969/j.issn.1007-2276.2013.12.004. 
[16] Cui L, Zhao XL, Li TH, et al. (2010). Athermalization of uncooled infrared optical system without focusing mechanism. Infrared Technology 32(4): 187-190. https://doi.org/10.3969/j.issn.1001-8891.2010.04.001.

[17] Zaoui FZ, Hanifi HA, Abderahman LY, Mustapha MH, Abdelouahed T, Djamel O. (2017). Free vibration analysis of functionally graded beams using a higherorder shear deformation theory. Mathematical Modelling of Engineering Problems 4(1): 7-12. https://doi.org/10.18280/mmep.040102.

[18] Medina YC, Khandy NH, Fonticiella OMC, Morales OFG. (2017). Abstract of heat transfer coefficient modelation in single-phase systems inside pipes. Mathematical Modelling of Engineering Problems 4(3): 126-131. https://doi.org/10.18280/mmep.040303.

[19] Maouassi A, Baghidja A, Daoud S, Zeraibi N. (2017). Numerical study of nanofluid heat transfer $\mathrm{Si} O 2$ through a solar flat plate collector. International Journal of Heat and Technology 35(1): 619-625. https://doi.org/10.18280/ijht.350319.

[20] Sarma PK, Konijeti R, Subramanyam T, Prasad LSV, Korada VS, Srinivas V, Vedula DR, Prasad VSRK. (2017). Fouling and its effect on the thermal performance of heat exchanger tubes. International Journal of Heat and Technology 35(1): 509-519. https://doi.org/10.18280/ijht.350307.

[21] Slătineanu L, Dodun O, Coteaţă M, Nagîț G, Beşliu I. (2016). Establishing requirements for mechanical equipment design. Academic Journal of Manufacturing Engineering 14(4): 106-111.

[22] Wang Y. (2017). Effect of different micro structures on heat dissipation of cpu surface heat sink. Journal of Manufacturing Engineering 15(3): 29-34. 